\title{
Mind Perception and Social Robots: The Role of Agent Appearance and Action Types
}

\author{
Imge Saltik \\ Interdisciplinary Neuroscience Program \\ Bilkent University, Turkey \\ imge.saltik@bilkent.edu.tr
}

\author{
Deniz Erdil \\ Department of Psychology \\ Bilkent University, Turkey \\ deniz.erdil@ug.bilkent.edu.tr
}

\author{
Burcu A. Urgen \\ Department of Psychology \& Interdisciplinary Neuroscience Program \\ National Magnetic Resonance Research Center (UMRAM) \\ Aysel Sabuncu Brain Research Center, Bilkent University, Turkey \\ burcu.urgen@bilkent.edu.tr
}

\begin{abstract}
Mind perception is considered to be the ability to attribute mental states to non-human beings. As social robots increasingly become part of our lives, one important question for HRI is to what extent we attribute mental states to these agents and the conditions under which we do so. In the present study, we investigated the effect of appearance and the type of action a robot performs on mind perception. Participants rated videos of two robots in different appearances (one metallic, the other human-like), each of which performed four different actions (manipulating an object, verbal communication, non-verbal communication, and an action that depicts a biological need) on Agency and Experience dimensions. Our results show that the type of action that the robot performs affects the Agency scores. When the robot performs human-specific actions such as communicative actions or an action that depicts a biological need, it is rated to have more agency than when it performs a manipulative action. On the other hand, the appearance of the robot did not have any effect on the Agency or the Experience scores. Overall, our study suggests that the behavioral skills we build into social robots could be quite important in the extent we attribute mental states to them.
\end{abstract}

\section{CCS CONCEPTS}

- Human-centered computing • Human-computer interaction (HCI) - HCI design and evaluation methods $\bullet$ User studies

KEYWORDS: Mind perception, Action perception, Human-like appearance, Human-Robot Interaction, Social robots, Robot design

\footnotetext{
Permission to make digital or hard copies of all or part of this work for personal or classroom use is granted without fee provided that copies are not made or distributed for profit or commercial advantage and that copies bear this notice and the full citation on the first page. Copyrights for components of this work owned by others than ACM must be honored. Abstracting with credit is permitted. To copy otherwise, or republish, to post on servers or to redistribute to lists, requires prior specific permission and/or a fee. Request permissions from Permissions@acm.org. HRI '21 Companion, March 8-11, 2021, Boulder, CO, USA.

(C) 2021 Association of Computing Machinery.

ACM ISBN 978-1-4503-8290-8/21/03...\$15.00.

https://doi.org/10.1145/3434074.3447161
}

ACM Reference format:

Imge Saltik, Deniz Erdil, Burcu A. Urgen. 2021. Mind perception and social robots: The role of agent appearance and action types. In Companion of the 2021 ACM/IEEE International Conference on Human-Robot Interaction (HRI'21 Companion), March 8-11, 2021, Boulder, CO, USA. ACM, New York, NY, USA. 5 pages. https://doi.org/10.1145/3434074.3447161

\section{Mind Perception and Social Robots}

Humans are social beings and they interact with other individuals in their environment such as other humans and animals. The remarkable advances in technology over the last century have given rise to a new kind of interaction which is between humans and social robots. Social robots are increasingly being used in a wide range of domains including education, patient care, rehabilitation, banks, airports, and entertainment [1]. How these new "individuals" are perceived by people, and whether they are accepted is an issue that has just begun to be addressed in humanrobot interaction research.

Studying how humans perceive and interact with robots is important for two reasons. First, robot design attempts without user feedback can result in robots that are not welcomed or accepted by humans. Thus, taking a human-centered approach in design [2] can guide engineers to build robots that are ergonomic and well-suited to their application domains. Second, understanding how humans perceive and interact with robots can teach us what makes us human $[3,4,5]$. Social robots are recent agents in our history and we have not evolved with them over many generations. Studying the interaction between humans and robots offers us tremendous opportunities in understanding human social cognition and enables us to re-evaluate our perception of our own species.

One of the most widely asked questions with the rise of artificial intelligence in general and robots in particular is whether humans perceive robots as having minds, a concept known as mind perception. Mind perception is not specific for robots but rather 
construed as a concept for any non-human being that is considered to have a mind. [6] suggest that we perceive mind in other things to understand, predict and control their behavior, and to establish a social bond with them. They also suggest that as a consequence of this, our interactions with them can become more effective, we may more likely exhibit socially accepted behaviors, and seek morality in those agents [6].

Gray and colleagues [7] conducted a comprehensive study to reveal dimensions of mind perception. This study showed that mind perception has two dimensions: Agency - the capacity to do, and Experience - the capacity to feel. The capacities that define agency are considered to be self-control, morality, memory, emotion recognition, planning, communication, and thinking. On the other hand, the capacities that define experience are considered to be hunger, fear, pain, pleasure, anger, desire, personality, consciousness, pride, shame, and fun.

There are few studies that study mind perception induced by robots by considering the framework of [7], in particular by measuring agency and experience dimensions. These studies have started to reveal the factors that modulate mind perception and its dimensions. One such factor is the physical appearance of robots. [8] found that robots with a more human-like appearance were perceived to have greater mental capacity, especially in the experience dimension, than the non-human-like ones. These results have been corroborated with a number of other studies that investigated the effect of appearance on mind perception using measures different from [7] such as theory of mind tasks [9] or self-reports in which people were directly asked whether they perceive a mind in the robot agents $[10,11]$. Among the studies that used the mind perception framework of [7], [12] reported that the behavior patterns of robots is another factor which affects mind perception induced by robots. They found that robots exhibiting unpredictable behavior were attributed more mental capacity than robots exhibiting predicted behavior. In addition, [13] showed that the social context of the robot affects the degree people attribute mental states to the robot.

All these studies have been informative to understand the conditions under which robots are attributed mental states but further work is needed considering the many scenarios in which we will be interacting with robots in our daily lives. We live in a dynamic world and as a consequence of this, one factor that can potentially influence the degree we attribute mental states to robots is the type of action they perform. For instance, one might think that a robot that communicates with a person has more mental capacity than a robot that does cleaning. Although action perception is a well-studied domain in psychology and neuroscience [14], including the study of robot actions [14-22], there is no study to the best of our knowledge that links action perception to attribution of mental states to robots in the framework of [7], considering the agency and experience dimensions.

In the present study, we manipulated the type of action the robot performs and measured mind perception. We hypothesized that robots that perform actions that are communicative in nature (verbal or non-verbal) or that are expected exclusively from biological beings would be rated to have more mental capacity than robots that perform actions that are manipulative in nature. We also manipulated the appearance of the agent to find out whether the appearance and the type of action can interact in mind perception.

\section{Methods}

\subsection{Participants}

76 university students participated in this study (Age: 18-35, Mean Age $=22.6, \mathrm{SD}=3.7 ; 50$ Female). All participants had normal vision. Before the experiment, all subjects signed a consent form approved by the Human Research Ethics Committee of Bilkent University.

\subsection{Stimuli}

The visual stimuli consisted of eight 2 -sec videos depicting 2 agents (Robot and Android), performing 4 actions (Wiping, Handwaving, Drinking, Introducing herself), taken from Saygin \& Ishiguro Robot Database, which were previously used in a number of studies [18-24].
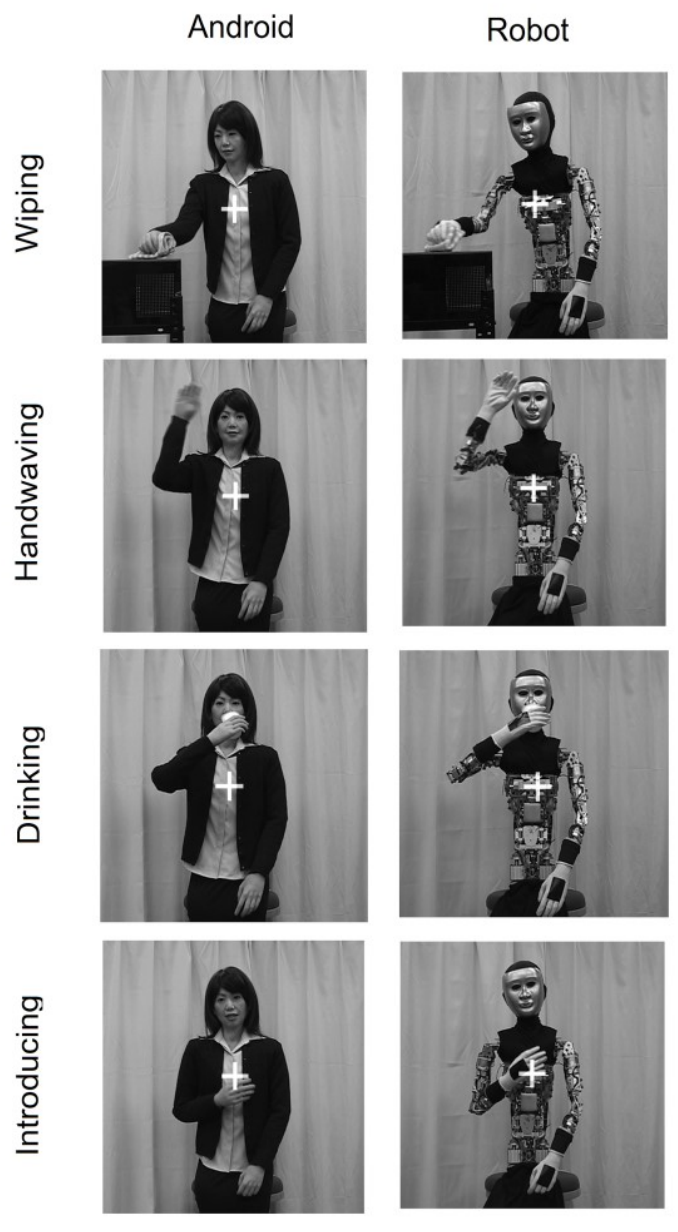

Figure 1: Static frames from the action videos used in the present study. 
Robot and Android agents were two different appearances of the Repliee Q2 robot, Robot having a metallic appearance, and Android having a human-like appearance. There is an artificial material imitating the human skin and human clothing on the Android whereas the Robot had metallic appearance without the clothes.

\subsection{Design}

There were two independent variables in the study, agent and action. Agent was a between-subject variable and has 2 levels: Robot (with metallic appearance) and Android (with human-like appearance). Action was a within-subject variable and has 4 levels: Wiping, Handwaving, Drinking, and Introducing herself. These actions were chosen as representatives of non-verbal communication (Handwaving), verbal communication (Introducing self), manipulation of an object (Wiping), biological needs (Drinking). Participants were randomly assigned to two groups, Robot group and Android group. Each group was shown all actions performed by the robot or the android.

The dependent variable in the study was mind perception, which was measured by using a questionnaire that has Agency and Experience components. The questions were prepared on the basis of the Mind Perception Questionnaire developed by [7, 25, 26]. There were 12 questions, half of which measure the Agency dimension and the other half measuring the Experience dimension. For Agency, the questions were about morality, emotion recognition, self-control, memory, planning and communication, and for Experience, the questions were about pleasure, fear, hunger, pain, anger, and desire as specified by [7]. The questions were shown in random order and the participants were asked to rate each video using a 7-point Likert scale ("0 Never" to" 6 - Ever"). (For questions see Appendix).

\subsection{Procedure}

Participants were divided into two groups: the first group were shown the Robot videos (50 people) and the second group were shown the Android videos (26 people). They were seated in front of a computer in a lab environment. Each group was shown a total of 48 questions (4 action videos x 12 mind perception questions). The questions were shown in random order for each participant.

\subsection{Data Analysis}

We computed two scores for each video: The Agency Score which is the average rating of the questions that measured the agency dimension, and the Experience Score which is the average rating of the questions that measured the experience dimension. We ran a 2 (Agent) x 4 (Action) mixed ANOVA on Agency scores and Experience scores.

\section{Results}

For Agency scores, we found a main effect of action $(\mathrm{F}(1,74)=$ 5.76, $\mathrm{p}<.001, \eta^{2}=0.012$ ). Post-hoc tests (Bonferroni corrected) revealed that participants rated the wiping action significantly lower than the other three actions - self introducing $(\mathrm{t}=-3.491, \mathrm{p}<$ $.003,95 \%$ CI $[-0.58,-0.08])$, drinking water $(\mathrm{t}=-3.106, \mathrm{p}<.013,95 \%$ $\mathrm{CI}[-0.54,-0.04])$ and hand waving $(\mathrm{t}=-3.523, \mathrm{p}<.003,95 \%$ CI $[-$
$0.58,-0.08]$ ) (Figure 2). However, there was not an effect of agent or interaction of agent and action.

For Experience scores, there was no effect of agent, action, or interaction of agent and action (Figure 3).

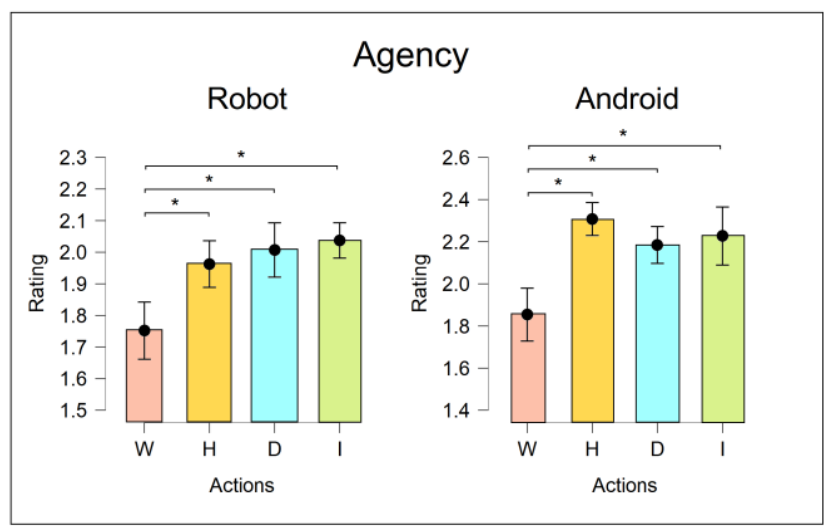

Figure 2: Agency scores from the study. Wiping (W) action was rated significantly lower than all other actions (Handwaving (H), Drinking (D), and Introducing self (I)) in agency dimension.

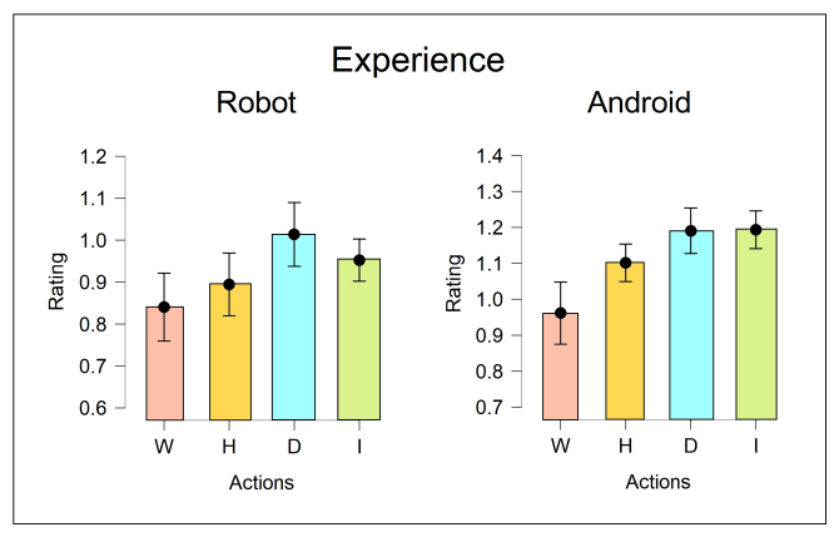

Figure 3: Experience scores from the study. All actions and agents were rated similarly in the experience dimension (W: Wiping, H: Handwaving, D: Drinking, I: Introducing self).

\section{Discussion}

In the present study, we investigated whether the type of action performed by robots and the robots' appearance (whether it is human-like or not) affect mind perception. To this end, we conducted a study in which participants were shown short videos of two robots, one human-like and the other metallic. Each robot performed four different actions that could be classified as nonverbal communication (hand waving), verbal communication (introducing self), manipulation (wiping a table), and biological need (drinking). Participants rated each video on a 7-point likert scale on questions that measured two dimensions of mind perception, Agency and Experience [7]. Our results showed that the type of action that the robot performs affects mind perception. When the robot performs an action that is communicative in nature or that depicts a biological need, it is rated higher in terms of having a mind compared to when it performs an action that is 
manipulative in nature (e.g. interacting with a physical object). This effect is present only on the Agency scores.

Our results extend previous work that investigates under what conditions humans attribute mental states to robots. Earlier work has shown that robots performing unpredictable behavior are rated higher to have a mind compared to robots that perform predictable behavior [12]. While our study did not specifically manipulate the "predictability" aspect of actions, our results are consistent with the findings of [12]. The actions that were rated higher in the present study were drinking, handwaving, and selfintroducing, all of which could be considered to be human-specific, and thus "unpredictable" when performed by a robot. In this respect, our study confirms and expands previous work by testing ecologically valid actions (instead of using images with description to actions) and revealing what kinds of actions can be valid in attributing mental states to robots.

It is important to note that although the type of action that the robot performs is important for mind perception scores, it affects only the agency dimension, and not the experience dimension. This suggests that the more human-specific actions a robot performs, the higher "the capacity to do" it will be attributed. However, no matter what type of action the robot performs, it is not considered to have a "capacity to feel". Of course, our study is limited in the number of actions varied as we were constrained with the type of actions Repliee Q2 robot can perform. Future work should investigate the effect of more complex and even affective actions on mind perception scores, especially on the experience dimension.

One surprising finding of our study was the lack of evidence for the effect of robot appearance on mind perception. This result is inconsistent with previous work that found that the more humanlike a robot is, the more mental capacity it is attributed, especially in the experience dimension [8]. There may be two explanations for this discrepancy. The first is the type of stimuli used in the two studies. In the present study, we used videos that depict the actions of two robots in different appearances, whereas in [8], static pictures were used. It is possible that videos provided a higher level of reality and ecological validity with their dynamic nature than static pictures. Therefore, regardless of the level of human-likeness, the two robots in the present study were rated similarly. A second explanation is the unbalanced number of participants in each group in the present study (Robot group vs. Android group). Although there were enough participants in each group to make a sound statistical argument, it is possible that the difference in the number of participants made a difference. We plan to collect more data to balance the number of participants.

The issue of ecological validity can be very important in understanding some of the other differences between our study and previous studies. Contrary to our research, there is evidence that people attribute mental states to robots when they share the same physical space with robots even if the robot does not have a human-like appearance [27 28, 29]. All these results show that more systematic studies are needed to understand the relationship between appearance, physical presence and mind perception.
Our study provides important insights for robot design. It seems like designing robots that can perform human-specific actions such as communicative actions or actions that depict biological needs could help people attribute more mental capacity to these agents. On the other hand, actions that are performed even by very simple robots that lack human form, such as cleaning are not considered to reflect mental states as much as human-specific actions. This suggests that the type of behavioral skills we build into robots will be crucial in how people perceive and interact with them. Knowing that the agent we are interacting with has a capacity to do many things we do or to feel like us will improve our interaction with them. We will probably better predict their behavior [30,31], interact with them more efficiently, may more likely exhibit socially accepted behaviors around them, and seek morality in those agents [6].

Our study also provides important insights about what makes us human. The ability to perform human-specific or biological actions is found to be an important sign of having agency. However, it does not seem to be sufficient to attribute a capacity to feel. This suggests that what makes us uniquely human is our capacity of doing a wide range of actions including the ones that are specific to our species and our capacity to experience and feel. Unless robots are equipped with these skills, it may not be likely that they would be attributed mental capacities as much as humans despite their perfect human-like appearances.

\section{ACKNOWLEDGMENTS}

We thank Irem Nur Özsoy for help in data collection.

\section{Appendix}

Mind Perception Questions:

Experience Questions (7-point Likert):

1. Pleasure: This robot can feel pleasure.

2. Fear: If this robot saw a snake, [it] would be scared.

3. Hunger: If this robot has not eaten breakfast, [it] may feel hungry.

4. Pain: If I squeezed the arm of this robot, [it] might feel pain.

5. Angry: This robot can get angry at something.

6. Desire: This robot can desire something.

Agency Questions (7-point Likert):

7. Morality: This robot can know the difference between good and bad actions.

8. Emotion recognition: If this robot sees someone happy/sad, [it] can understand these feelings.

9. Self-control: If this robot moves, [it] chooses to move.

10. Memory: This robot is capable of remembering.

11. Planning: This robot can plan things.

12. Communication: If contacted with this robot, [it] responds.

Note: This task was adapted from studies Gray et al., 2007 [7]; Brink et al. [25], 2019; Malle, 2019 [26] by the authors.

\section{REFERENCES}

[1] Elizabeth Broadbent. 2017. Interactions with robots: The truths we reveal about ourselves. Annual review of psychology, 68, 627-652.

[2] Donald A. Norman. 1988. The psychology of everyday things. Basic books.

[3] Karl MacDorman and Hiroshi Ishiguro. 2006. The uncanny advantage of using androids in cognitive and social science research. Interaction Studies, 7, 297337. 
[4] Ayse Pinar Saygin, Thierry Chaminade, Burcu Aysen Urgen and Hiroshi Ishiguro. 2011. Cognitive neuroscience and robotics: a mutually beneficial joining of forces in Robotics: Systems and Science, ed. L. Takayama (Los Angeles: MIT Press).

[5] Emily S. Cross, Ruud Hortensius and Agnieszka Wykowska. 2019. From social brains to social robots: Applying neurocognitive insights to human-robot interaction. Philos. Trans. R. Soc. B Biol. Sci., 374, 5-8.

[6] Adam Waytz, Kurt Gray, Nicholas Epley, Daniel M. Wegner. 2010. Causes and consequences of mind perception. Trends Cogn. Sci. 14, 383-88.

[7] Heather M. Gray, Kurt Gray and Daniel M. Wegner. 2007. Dimensions of mind perception. Science 315:619

[8] Kurt Gray and Daniel M. Wegner. 2012. Feeling robots and human zombies: mind perception and the uncanny valley. Cognition $125, \quad 125-130$.

[9] Jaime Banks. 2020. Theory of mind in social robots: Replication of five established human tests. International Journal of Social Robotics, 1-12.

[10] Abdulaziz Abubshait \& Eva Wiese. 2017. You look human, but act like a machine: agent appearance and behavior modulate different aspects of humanrobot interaction. Frontiers in psychology, 8, 1393.

[11] Molly C. Martini, Christian A. Gonzalez, Eva Wiese. 2016. Seeing minds in others-Can agents with robotic appearance have human-like preferences? PloS one, 11(1), e0146310.

[12] Adam Waytz, Carey Morewedge, Nicholas Epley, George Monteleone, Jia-Hong Gao, and John T. Cacioppo. 2010. Making sense by making sentient: Effectance motivation increases anthropomorphism. Journal of Personality and Socia Psychology, 99(3), 410-435.

[13] Rebecca Butler, Zoe Pruitt and Eva Wiese. 2019. The Effect of Social Context on the Mind Perception of Robots. Proceedings of the Human Factors and Ergonomics Society Annual Meeting, 63(1), 230-234.

[14] Svenja Caspers, Karl Zilles, Angela R.Laird, Simon B.Eickhoff. 2010. ALE meta-analysis of action observation and imitation in the human brain Neuroimage, 50(3), 1148-1167.

[15] Valeria Gazzola, Giacomo Rizzolatti, B. Wicker, Christian Keysers. 2007. The anthropomorphic brain: the mirror neuron system responds to human and robotic actions. Neuroimage, 35(4), 1674-1684.

[16] Lindsay M.Oberman, Joseph P.McCleery, Vilayanur S.Ramachandran, Jaime A.Pineda. 2007. EEG evidence for mirror neuron activity during the observation of human and robot actions: Toward an analysis of the human qualities of interactive robots. Neurocomputing, 70(13-15), 2194-2203.

[17] Emily S. Cross, Roman Liepelt, Antonia F. de C. Hamilton, Jim Parkinson, Richard Ramsey, Waltraud Stadler, and Wolfgang Prinz. 2012. Robotic movement preferentially engages the action observation network. Human brain mapping, 33(9), 2238-2254.
[18] Ayse Pinar Saygin, Thierry Chaminade, Hiroshi Ishiguro, Jon Driver and Chris Frith. 2012. The thing that should not be: predictive coding and the uncanny valley in perceiving human and humanoid robot actions. Social Cognitive Affective Neuroscience, 7, 413-422.

[19] Burcu Aysen Urgen, Markus Plank, Hiroshi Ishiguro, Howard Poizner and Ayse Pinar Saygin. 2013. EEG theta and mu oscillations during perception of human and robot actions. Frontiers in Neurorobotics, 7-19.

[20] Burcu Aysen Urgen, Marta Kutas, Ayse Pinar Saygin. 2018. Uncanny valley as a window into predictive processing in the social brain. Neuropsychologia, 114, 181-185.

[21] Burcu Aysen Urgen, Selen Pehlivan, Ayse Pinar Saygin 2019. Distinct representations in occipito-temporal, parietal, and premotor cortex during action perception revealed by fMRI and computational modeling. Neuropsychologia, 127, 35-47.

[22] Burcu Aysen Urgen, Ayse Pinar Saygin. 2020. Predictive processing account of action perception: Evidence from effective connectivity in the Action Observation Network. Cortex.

[23] Busra Sarigul, Imge Saltik, Batuhan Hokelek, \& Burcu Aysen Urgen 2020. Does the Appearance of an Agent Affect How We Perceive his/her Voice? Audiovisual Predictive Processes in Human-robot Interaction. In Companion of the 2020 March ACM/IEEE International Conference on Human - Robot Interaction. 430-432.

[24] Burcu A. Urgen, İlayda Guneysu, Selin Yilmaz, Begum Cerrahoglu and Ece Dincer. 2020. Do Robots Distract us as much as Humans? The Effect of Humanlike Appearance and Perceptual Load. In Companion of the 2020 March ACM/IEEE International Conference on Human - Robot Interaction. 493-495.

[25] Kimberly A. Brink, Kurt Gray and Henry M. Wellman. 2019.Creepiness creeps in: Uncanny valley feelings are acquired in childhood.Child development, 90(4) 1202-1214.

[26] Bertram Malle. 2019. How many dimensions of mind perception really are there? In CogSci pp. 2268-2274.

[27] Wesley Buckwalter \& Mark Phelan. 2013. Function and feeling machines: a defense of the philosophical conception of subjective experience. Philosophical Studies, 166(2), 349-361.

[28] Brian Fiala, Adam Arico, \& Shaun Nichols. 2014. You, robot.

[29] Justin Sytsma, Edouard Machery. 2010. Two conceptions of subjective experience. Philosophical studies, 151(2), 299-327.

[30] Yuto Imamura, Kazunori Terada, Hideyuki Takahashi. 2015. Effects of behavioral complexity on intention attribution to robots. In Proceedings of the 3rd International Conference on Human-Agent Interaction pp. 65-72.

[31] Sam Thellman, Tom Ziemke. 2020. Do You See what I See? Tracking the Perceptual Beliefs of Robots. Iscience, 23(10), 101625 